\title{
La evaluación del desarrollo pragmático temprano a través del EDPRA y The Pragmatics Profile
}

\author{
The assessment of early pragmatic development by mean of EDPRA and The Pragmatics Profile \\ Iria Botana , Manuel Peralbo \\ Centro Atención Temprana Ordes (A Coruña) Departamento de Psicología Evolutiva, Universidad de la Coruña
}

\begin{abstract}
Resumen
El objetivo del presente trabajo es diseñar un instrumento de evaluación en lengua española, que permita analizar el uso comunicativo del lenguaje y obtener un perfil pragmático en edades tempranas .

Partiendo del trabajo de Dewart y Summers The pragmatics profile (1995) e incluyendo las funciones descritas por Halliday (1975), se elaboró una escala de desarrollo pragmático denominada EDPRA.

En la aplicación inicial participaron un total de 30 niños y niñas de edades comprendidas entre los 6 y los 48 meses. Se evaluaron mediante el EDPRA y The Pragmatic Profile (1995). Los resultados muestran una alta fiabilidad y validez del EDPRA comparado con el Pragmatics Profile. La ampliación de la muestra permitirá elaborar baremos y perfiles útiles para la evaluación temprana de estas funciones comunicativas.
\end{abstract}

Palabras clave: desarrollo pragmático, intervención temprana, evaluación del desarrollo del lenguaje.

\begin{abstract}
The objective of the present study is design an assessment tool in Spanish language, allowing analyzer the communicative use of language and gets a pragmatic profile at early ages. Based on work by Dewart and Summers The pragmatics profile (1995) and including the functions described by Halliday (1975), developed a scale of pragmatic development called EDPRA. A total of 30 boys and girls aged between 6 and 48 months participated in the initial application. They were evaluated by the EDPRA and The Pragmatic Profile (1995). The results show a high reliability and validity of the EDPRA compared to the Pragmatics Profile. The expansion of the sample will allow developing scales and profiles useful for early evaluation of these communicative functions.
\end{abstract}

Keywords: pragmatic development, early intervention, language development assessment.

\section{Introducción}

En el marco de la atención temprana, en particular en el campo de la intervención logopédica, los instrumentos de evaluación nos han permitido aproximarnos al conocimiento del desarrollo infantil y poder así valorar de forma más precisa a niños de menor edad, lo que favorece la prevención y el planteamiento de programas de intervención más precoces y eficaces. Aún así, mantenemos un déficit significativo en cuanto a escalas del área comunicativa para niños menores de cuatro años, en lengua castellana, poseemos instrumentos que permiten evaluar aspectos formales del lenguaje, vocabulario, marcadores morfosintácticos e incluso comprensión del mismo. Pero todavía no disponemos de instrumentos estandarizados para evaluar el uso comunicativo $\mathrm{y}$ obtener un perfil pragmático en edades tempranas. En la práctica clínica los cuestionarios parentales ofrecen información de gran relevancia y junto con los protocolos de observación aportan calidad y valor clínico a la valoración de la comunicación en el niño pequeño, más, si queremos completar la valoración de forma rigurosa, es necesario la aplicación de escalas estandarizadas que nos permita obtener puntuaciones típicas e interpretar las mismas en referencia a la población española.

Para poder analizar el papel de la pragmática en la evaluación del lenguaje infantil debemos recoger algunos de los autores más relevantes en el enfoque pragmático. Desde que Morris (1972) introdujo el término pragmática como una de las ramas de investigación de la semiótica, junto a la sintaxis y a la semántica, definiéndola como el estudio de la relación de los signos con sus intérpretes, se han producido numerosas definiciones del término, Crystal (2003) define pragmática, como el estudio del lenguaje desde el punto de vista de los usuarios, especialmente de las elecciones que realizan, los problemas que encuentran al usar la lengua en la interacción social y los efectos que el uso de la lengua tiene en otros participantes en el acto comunicativo.

Diversos autores defienden el lugar que debe ocupar la pragmática cuando hablamos de evaluación, tal y como se recoge en el trabajo de Acuña \& Snetis (2004) desde el punto de vista pragmático, el desarrollo del niño se caracteriza como un proceso en el que se manifiesta una cantidad de actividades eminentemente sociales y comunicativas y su evaluación permite conocer no solo datos sobre su el desarrollo del lenguaje sinó también sobre su desarrollo social.

Desde la perspectiva teórica propuesta por Halliday (1975) se identifican entre los nueve y los dieciocho meses seis funciones pragmáticas. En esta línea se encuentran también los trabajos de Dale (1980) en los que ofrece categorías de análisis para la valoración del status del diálogo que correlacionan con funciones pragmáticas. 
A lo largo de las últimas tres décadas del siglo pasado y la primera del siglo XXI han ido apareciendo propuestas elaboradas por investigadores de diferentes países, orientadas a mejorar las habilidades comunicativas y lingüísticas de los niños que presentan trastornos del lenguaje asociados o no a algún tipo de discapacidad. Las diferentes propuestas de intervenciones de corte naturalista surgen paralelamente al enfoque pragmático, acompañadas por el modelo interactivo y transaccional de adquisición del lenguaje basado en la observación del contexto natural de las interacciones de los niños. Son modelos y teorías que enfatizan el valor del uso y las funciones del lenguaje frente al predominio de los aspectos más formales. Desde esta perspectiva ecológica cobra mayor peso la evaluación de la interacción y uso del lenguaje respecto a la forma. Ello justifica la necesidad de mejoras en los instrumentos de evaluación de la pragmática.

Tenemos por otro lado las aportaciones desde el campo de la lingüística clínica más reciente la defensa del enfoque pragmático para el diseño y proyección de pruebas de evaluación lingüística en edad infantil, sustentando que el único modo de reconocer y comprobar las lenguas es en su uso, en la vertiente pragmática y de interacción: seráen las coordenadas en donde se observen posibilidades y se detecten inconvenientes (Fernández, 2000). La lingüística clínica ha permitido además, realizar una clasificación de la pragmática en su dimensión lócutiva , tenemos en esta línea los trabajos de Gallardo (1996) que proponen categorizar las habilidades pragmáticas postlocutivas en tres grandes bloques la pragmática enunciativa, la pragmática textual y la pragmática interactiva.

Décadas atrás, Belinchon (1985) recogía al detalle la escasez de trabajos dedicados específicamente a la evaluación de las funciones pragmáticas del lenguaje en la primera infancia, a sú vez hacía referencia al análisis de las posibles alternativas metodológicas que podrían utilizarse en el estudio de la adquisición pragmática del lenguaje y la complejidad de las mismas. a pesar de las lineas y sugerencias de las investigaciones, décadas después, en el 2015, no disponemos de instrumentos de evaluación de las funciones pragmáticas en niños menores de 4 años.

El objetivo principal de este trabajo fue comprobar si la escala de aplicación directa (EDPRA) que se elaboró para la evaluación de la pragmática en niños menores de 4 años evalúa las dimensiones recogidas en el cuestionario parental The Pragmátics Profile de Dewarts y Summers( 1995).

\section{Participantes}

El muestreo se realizó siguiendo un criterio de conveniencia. Como criterio de exclusión se descartaron aquellos casos en los que pudiera existir sospecha de una alteración que afecte al desarrollo comunicativo , lingüistico o cognitivo de los niños. Además, fueron considerados también criterios de exclusión la existencia de deficiencia audivita moderada o grave, deficiencia visual severa, síndrome de filiación genética, grandes prematuros,trastorno del neurodesarrollo,trastornos del sistema nervios central, hospitalización prolongada, institucionalización durante los primeros meses de vida.

La muestra fue estraída de dos centros de educación infantil públicos de la provincia de la Coruña ( ambos ubicados en la comarca de rdenes), siguiendo los protocolos de actuación pertinentes en materia de investigación en los que se incluyen los consentimientos informados de los padtres de los participantes así como de las propias escuelas infantiles.

El tamaño muestral requerido para el análisis pricométrico inicial fue $n=30,18$ niños y 12 niñas. La edad cronológica de los participantes oscilaba entre los 7 meses y los 42 meses .

\section{Instrumentos}

The Pragmatics Profile (Dewart y Summers, 1995)

Tras un análisis de los instrumentos de evaluación pragmática disponibles, recogimos cuestionarios parentales entre otros el Mcarthur (Fenson y cols, 2007), el protocolo pragmático de Prutting y Kirchner (1987), el protocolo de evaluación pragmátca de Martinez (2001), el infant/toddler checklist de Wetherby y Barry (2001), el CCC-2 de $\operatorname{Bishop}(2003)$ y the pragmátics profile de Dewart and Summers (1995). De todos ellos solo the pragmatics profile permite la evaluación de niños entre los 6 y los 48 meses, infant/toddler cheklist es para niños entre 6 y 24 meses y los demás están pensados para el analisis de la pragmática postlocutiva.

Además el Profile dispone de una versión Pre-scholar que coincide con las edades objeto de nuestro estudio (0-4años), esta versión fue traducida al castellano.

El profile es un cuestionario para padres que obtiene respuestas descriptivas de las conductas de sus hijos y no ofrece datos cuantificables por lo cual establecimos unas equivalencias entre las respuestas posibles y una puntuación o equivalencia numérica $(0,1,2)$ para el posible análisis estadísitco de los datos obtenidos en el mismo. Correspondiendose 0 con el nivel más bajo de adecuación de la conducta a la esperada y 2 con el nivel más alto de adecuación de la conducta esperada.

\section{Escala de Desarrollo Pragmático (EDPRA).}

La construcción de la escala EDPRA se encuentra en este momento en la fase de primeros análisis y estudio piloto , esta escala ha sido elaborada basandonos en el trabajo de Dewart y Summers(1995) y en la teoríade funciones comunicativas de Halliday ( 1985).

Para ello partimos de la estructura e hitos de desarrollo pragmático descritos en el Profile en su versión Pre-Scholar de 0 a 4 años. La estructura que sigue el Profile se basa en cuatro ejes de evaluación:

A- Funciones comunicativas

B- Respuesta a la comunicación

C- Interacción en la conversación

D- Variación contextual

Se mantuvo la misma estructura en la que consideran las tres áreas primeras como fundamentales del desarrollo de la pragmática, no así la variación contextual, que aunque sí se incluye en el Profile, durante la descripción de la prueba se considera complementaria. Por este motivo y debido al objetivo de nuestro trabajo que implica la valoración pragmática a través de un test aplicado al propio niño en un único contexto, incluir 
más contextos de evaluación dificulta uno de los objetivos de nuestro trabajo, que es la aplicación directa de del terapeuta.

Realizamos una traducción y adaptación al español y posteriormente contruimos una escala de desarrollo pragmático denominada EDPRA con tres ejes de evaluación pragmática . Cada eje incluye un máximo de 6 items cuantificables con puntuaciones de $0,1,2$ ó 3 . Al mismo tiempo hemos descrito de manera detallada una situación que favorece la realización del ítem.

La escala EDPRA dispone de una hoja de respuesta con sus correspondientes ítems para cada rango de edad: 6 a 12 meses, 12 a 24 meses, 24 a 36 meses y 36 a 48 meses. Todas ellas tienen un máximo de 18 ítems descritos a partir de situaciones conocidas para los niños.

\section{Resultados}

Los análisis estadísticos se realizaron a través del SPSS v19.0.

En primer lugar, se realizó un contraste no paramétrico, U de Mann-Whitney, para determinar si existían diferencias debidas al sexo en los resultados de ambas pruebas. En este sentido, no se obtuvieron resultados significativos. Es decir niñas y niños puntúan de forma equiparable en cuanto a desarrollo pragmático tanto en el EDPRA como en el Pragmatics Profile.

A Continuación, mediante la prueba Chi-cuadrado, se analizaron las asociaciones entre el grupo de edad y funciones del EDPRA y el Profile, en este caso se obtuvieron resultados significativos en el EDPRA A funciones comunicativas $(X(30)=22,80, p=.012)$ y el eje de respuesta comunicativa del Profile $(X(30)=40,00$, $p<.001)$.

Analizando mediante la prueba U de Mann-Whitney la asociación entre el grupo de edad y los ejes del EDPRA y el Profile se han obtenido los siguientes resultados significativos:

1. Entre el grupo de 6 a 12 meses y el grupo de 12 a 24 meses el EDPRA C ( $z=-1.964, p 0.05)$ y los ejes $\mathrm{A}(z=-2.243, p 0.05), \mathrm{B}(z=-2.634, p 0.05)$ y $\mathrm{C}(z=-$ $2.492, p$ 0.05) del Profile existen diferencias significativas.

2. Al comparar el grupo de 6 a 12 meses y el de 24 a 36 meses aparecen diferencias significativas en EDPRA B $(z=-2.032, \mathrm{p} 0.05)$ y C $(z=-2.364, p 0.05)$ y todos los ejes del Profile Profile A $(z=-2.343, p$ 0.05), Profile $\mathrm{B}(z=-2.343, p 0.05)$, y Profile C $(z=-2.449$, $p$ 0.05).

3. Al comparar el grupo de 6 a 12 meses y el de 36 a 48 meses aparecen diferencias significativas en EDPRA B ( $\mathrm{z}=-2.564, p$ 0.05). y C ( $z=-2.593, p 0.05)$. $\mathrm{y}$ todos los ejes del Profile Profile A ( $\mathrm{z}=-2.550$, $p$ 0.05), Porfile B ( $z=-2.790, p 0.05)$, y Profile C ( $z=$ 2.593, $p$ 0.05).

4. Entre los grupos de edad de 12 a 24 meses y 36 a 48 meses existen diferencias significativas en todos los ejes del EDPRA: EDPRA A $(z=-2, p$ 0.05). y los ejes A y C del Profile.

5. En la comparativa de los grupos de 12 a 24 meses y 24 a 36 meses los resultados son significativos en todos los ejes del EDPRA EDPRA A $(z=-1.946$, $p 0.05)$ EDPRA B $(z=-3.434, \mathrm{p} 0.05)$ EDPRA C $(z=$
$2.779, p 0.05)$ y los ejes A y C del Profile, Profile A $(z=-2.685, p$ 0.05), Profile C ( $z=-2.873, p 0.05)$.

6. Entre los grupos 24 a 36 meses y 36 a 48 meses sólo hemos obtenido resultados significativos en el Profile B, $(z=-2.006, p$ 0.05)

Además, como se puede ver en la tabla 1, existe una correlación significativa entre la edad en meses y los ejes evaluados en el EDPRA y el Profile.

Tabla 1. Resultados de la correlación de Spearman entre edad en meses y las funciones comunicativas del EDPRA y Pragmatic Profile

\begin{tabular}{ll}
\hline Funciones comunicativas EDPRA & $r=.544$ \\
Respuesta comunicativa EDPRA & $r=.821$ \\
Interacción y Conversación EDPRA & $r=.784$ \\
Funciones comunicativas PROFILE & $r=.753$ \\
Respuesta comunicativa PROFILE & $r=.619$ \\
Interacción y Conversación PROFILE & $r=.869$ \\
\hline
\end{tabular}

A continuación, se trató de comprobar si entre ambas pruebas, EDPRA y Profile, existía una correlación alta y significativa que pudiera avalar la validez convergente de la primera.

Se ha obtenido una correlación altamente significativa entre ambas pruebas $\left(r_{s}=.573, \rho \quad .001\right)$

En la siguiente figura podemos ver la comparativa entre las puntuaciones obtenidas en cada una de las pruebas. Se observa una tendencia clara de aumento de las puntuaciones con la edad, además se puede ver paralelismo evidente en las líneas seguidas por sus ejes.

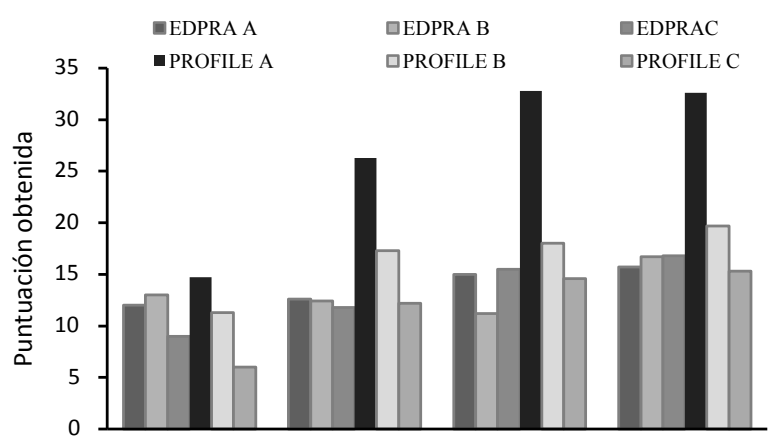

Figura 1: Perfiles del EDPRA y Profile en función de la edad

A excepción del EDPRA B, todas las pruebas obtienen resultados proporcionalmente similares entre sí. Las diferencias obtenidas en el EDPRA B pueden estar indicando diferencias en la sensibilidad de ambas pruebas.

\section{Discusión}

Los datos reflejan una alta correlación entre la prueba de aplicación directa elaborada, EDPRA, y el cuestionario parental The Pragmatics Profile de Dewart y Summers. Lo cual sugiere similitudes significativas entre ambas.

Los resultados iniciales sugieren que se trata de una medida válida de las habilidades del lenguaje pragmático de los niños sin alteraciones del desarrollo con edades entre los 6 y los 48 meses en el contexto de juego terapeuta-niño. Además el tiempo de aplicación del 
EDPRA es corto, aproximadamente 15-20 minutos. Esto es importante ya que el EDPRA pretende llegar a ser una herramienta clínica que ayude a los profesionales en la identificación fiable y en el seguimiento de los cambios clínicos en el tiempo.

Hay una clara necesidad en el campo de la evaluación del desarrollo pragmático. En este trabajo, hemos abogado por la creación de un instrumento que permitauna evaluación observacional del lenguaje pragmático a partir de contextos controlados próximos a las interacciones de juego naturalista, con un sistema de puntuación basado en la descripción detallada que disminuya el componente subjetivo que la observación implica.

El establecimiento de las propiedades psicométricas del EDPRA será el primer paso hacia su contribución a esta área descuidada, pero muy necesaria de la investigación.

La investigación futura podría también considerar la relación entre el EDPRA y los instrumentos que evalúan la socialización entre iguales en contextos naturalistas, como el Inventario Manchester por Zona de juegos Observación (MIPO: Gibson, Hussain, Holsgrove, Adams, y Green, 2011).

Sería necesario la ampliación de la muestra, así como un análisis factorial detallado, que permita conocer las propiedades psicométricas del EDPRA, analizar su distribución en ejes, su sistema de puntuación y profundizar en el análisis de su validez en la evaluación del desarrollo pragmático.

\section{Referencias}

Acuña, X., \&Sentis, F. (2004). Desarrollo pragmático en el habla infantil. Revisita Onomázein.10(2), 33-56.

Bates, E., McNew, S., MacWhinney, B., Devescovi, A.,\& Smith, S. (1982). Functional constraints on sentence processing: A cross-linguistic study. Cognition, 11(3),245-299. http://dx.doi.org.10.1016/0010-0277(82)90017-8

Bishop, D.V.M. (2003). The Children's Communication Checklist: CCC-2. London: Harcourt Assessment.
Carmona, M. B. (1984). Adquisición y evaluación de las funciones pragmáticas del lenguaje: un estudio evolutivo. Estudios de Psicología, 5(19-20), 35-49. http://dx.doi.org.10.1080/02109395.1984.10821400

Crystal, D. (2003). English as a Global Language. Cambridge: Cambridge University Press.

http://dx.doi:10.5785/20-1-80

Dale, P. (1980). Desarrollo del lenguaje. México: Trillas.

Dewart, H. y Summers, S. (1995).The pragmatics profile of everyday communication skills in children. NFERNelson.

Fernández Pérez, M. (2001). El enfoque pragmático en el diseño y proyección de pruebas de evaluación lingüística en edad infantil.Pragmalingüistica, 8-9,113-122.

Fenson, L., Marchman, V. A., Thal, D. J., Dale, P. S., Reznick, J. S., \& Bates, E. (n.d.).MacArthur-Bates Communicative Development Inventories, Second Edition. PsycTESTS Dataset.

http://dx.doi.org.10.1037/t11538-00

Gallardo, B. (1996). Análisis conversacional y pragmática del receptor. Episteme.

Halliday, M. A. (1975). Estructura y función del lenguaje. En John Lyons (ed.), Nuevos horizontes de la lingüística, Alianza Editorial, Madrid, 145-173

Martínez, L. (2001).Protocolo de Evaluación Pragmática. Escuela de Fonoaudiología. Facultad de Medicina Universidad de Chile.

Morris, C. (1972). Fundamentos de la teoría de los signos. En F.Gracia, Presentación del lenguaje. Madrid. Taurus, 53-65 ( $1^{\mathrm{a}}$ ed 1938$)$

Prutting, C. A., \&Kittchner, D. M. (1987). A Clinical Appraisal of the Pragmatic Aspects of Language.J Speech Hear Disord, 52(2), 105. http://dx.doi.org.10.1044/jshd.5202.105

Wetherby, A. M., \& Prizant, B. M. (n.d.). Communication and Symbolic Behavior Scales Developmental Profile, First Normed Edition. PsycTESTS Dataset. http://dx.doi.org.10.1037/t11529-000 